Mathematical Sciences and Applications

E-NOTES

MATHEMATICAL

SCIENCES

AND APPLICATIONS

E-NOTES

https://doi.org/10.36753/mathenot.692053

10 (2) 93-101 (2022) - Research Article

ISSN: 2147-6268

(c)MSAEN

\title{
Some Properties of Two Dimensional Interval Numbers
}

\author{
Fatih Nuray, Uğur Ulusu* and Erdinç Dündar
}

\begin{abstract}
In this paper, we will introduce the notion of convergence of two dimensional interval sequences and show that the set of all two dimensional interval numbers is a metric space. Also, some ordinary vector norms will be extended to the set of two dimensional interval vectors. Furthermore, we will give definitions of statistical convergence, statistically Cauchy and Cesàro summability for the two dimensional interval numbers and we will get the relationships between them.
\end{abstract}

Keywords: Convergent sequence; Metric spaces; Interval number sequence; Two dimensional interval number.

AMS Subject Classification (2020): Primary: 40C05 ; Secondary: 40J05; 46A45.

${ }^{*}$ Corresponding author

\section{Introduction}

It is known that many mathematical structures have been constructed with real or complex numbers. In recent years, these mathematical structures were replaced by interval numbers and these mathematical structures have been very popular for three decades.

In order fully and effectively to utilize pure mathematics for the analysis of natural phenomena, we must be aware that there are many phases concerning which mathematics and reality do not perfectly agree. For example neither one point on the real number axis is sufficient to represent a physical quantity, no any trace of a moving body described completely as a continuous function of time having no breadth. The concept of an interval is more fundamental than that of a real number. The concept of an interval is fundamental is not only in the case of numerical calculation. It is better to use an interval instead of real number. Interval arithmetic was first suggested by Dwyer [2]. Development of interval arithmetic as a formal system and evidence of its value as a computational device was provided by Moore [11] and Moore and Yang [12]. Furthermore, Moore and others [2, 3, 6, 7, 10] have developed applications to differential equations. Chiao [1] introduced sequence of interval numbers and defined usual convergence of sequences of interval numbers. Markov $[8,9]$ studied on interval arithmetic. Şengönül and Eryilmaz [15] introduced and studied convergent and bounded sequence spaces of interval numbers and showed that these spaces are complete metric space. The concept of a two dimensional interval is more fundamental than that of a one dimensional interval. In this paper, we introduce and study two dimensional interval sequences. 
The set of all $\xi=\left(\xi_{1}, \xi_{2}\right) \in \mathbb{R}^{2}$ satisfying the condition $\xi_{1 \underline{\underline{\ell}}} \leq \xi_{1} \leq \xi_{1 \underline{\underline{r}}}$ and $\xi_{2 \bar{\ell}} \leq \xi_{2} \leq \xi_{2 \bar{r}}$ is called the two dimensional interval (or two dimensional interval vector) and is denoted by $\left(\left[\xi_{1 \underline{\ell}}, \xi_{1 \underline{r}}\right],\left[\xi_{2 \bar{\ell}}, \xi_{2 \bar{r}}\right]\right)$. Let's denote the set of all closed two dimensional intervals by $\mathfrak{R}^{2}$. Any elements of $\mathfrak{R}^{2}$ are called a closed rectangle or closed two dimensional interval and it denoted by $\bar{\xi}$. That is

$$
\bar{\xi}=\left\{\left(\xi_{1}, \xi_{2}\right) \in \mathbb{R}^{2}: \xi_{1 \underline{\ell}} \leq \xi_{1} \leq \xi_{1 \underline{\underline{r}}} \text { and } \xi_{2 \bar{\ell}} \leq \xi_{2} \leq \xi_{2 \bar{r}}\right\} .
$$

For all $\bar{\xi}_{1}, \bar{\xi}_{2} \in \mathfrak{R}^{2}$, we have $\bar{\xi}_{1}=\bar{\xi}_{2}$ if and only if $\xi_{1 \underline{\underline{\ell}}}=\xi_{2 \underline{\underline{\ell}},} \xi_{1_{\underline{r}}}=\xi_{\underline{\underline{r}}^{\prime}}, \xi_{1 \bar{\ell}}=\xi_{2 \bar{\ell}}$ and $\xi_{1 \bar{r}}=\xi_{2 \bar{r}}$.

$$
\bar{\xi}_{1}+\bar{\xi}_{2}=\left\{\left(\xi_{1}, \xi_{2}\right) \in \mathbb{R}^{2}: \xi_{1 \underline{\ell}}+\xi_{2 \underline{\ell}} \leq \xi_{1} \leq \xi_{1 \bar{r}}+\xi_{2 \bar{r}}, \xi_{1 \bar{\ell}}+\xi_{2 \bar{\ell}} \leq \xi_{2} \leq \xi_{1 \bar{r}}+\xi_{2 \bar{r}}\right\} .
$$

If $\alpha>0$ then

$$
\alpha \bar{\xi}=\left\{\left(\xi_{1}, \xi_{2}\right) \in \mathbb{R}^{2}: \alpha \xi_{\underline{l}} \leq \xi_{1} \leq \alpha \xi_{\underline{r}} \text { and } \alpha \xi_{\bar{l}} \leq \xi_{2} \leq \alpha \xi_{\bar{r}}\right\}
$$

and $\alpha<0$ then

$$
\begin{aligned}
& \alpha \bar{\xi}=\left\{\left(\xi_{1}, \xi_{2}\right) \in \mathbb{R}^{2}: \alpha \xi_{\underline{r}} \leq \xi_{1} \leq \alpha \xi_{\underline{l}} \text { and } \alpha \xi_{\bar{r}} \leq \xi_{2} \leq \alpha \xi_{\bar{l}}\right\} \\
& \bar{\xi}_{1} \bar{\xi}_{2}=\left(\left[\min \left\{\xi_{1 \underline{\ell}} \xi_{2 \underline{\ell}}, \xi_{1 \underline{\underline{\ell}}} \xi_{2_{\underline{r}}}, \xi_{1_{\underline{r}}} \xi_{2_{\underline{r}}}, \xi_{1_{\underline{r}}} \xi_{2 \underline{\ell}}\right\}, \max \left\{\xi_{1 \underline{\underline{\ell}}} \xi_{2 \underline{\underline{\ell}}}, \xi_{1 \underline{\underline{\ell}}} \xi_{2_{\underline{r}}}, \xi_{1_{\underline{r}}} \xi_{2_{\underline{r}}}, \xi_{1_{\underline{r}}} \xi_{2 \underline{\ell}}\right\}\right],\right. \\
& \left.\left[\min \left\{\xi_{1 \bar{\ell}} \xi_{2 \bar{\ell}}, \xi_{1 \bar{\ell}} \xi_{2 \bar{r}}, \xi_{1 \bar{r}} \xi_{2 \bar{r}}, \xi_{1 \bar{r}} \xi_{2 \bar{\ell}}\right\}, \max \left\{\xi_{1 \bar{\ell}} \xi_{2 \bar{\ell}}, \xi_{1 \bar{\ell}} \xi_{2 r}, \xi_{1 \bar{r}} \xi_{2 \bar{r}}, \xi_{1 \bar{r}} \xi_{2 \bar{\ell}}\right\}\right]\right) .
\end{aligned}
$$

The absolute value of a two dimensional interval is defined by

$$
|\bar{\xi}|=\max \left\{\left|\xi_{\underline{\underline{\underline{ }}}}\right|,\left|\xi_{\underline{r}}\right|,\left|\xi_{\bar{\ell}}\right|,\left|\xi_{\bar{r}}\right|\right\} \text {. }
$$

\section{Main Results}

Theorem 2.1. The set of all two dimensional interval numbers $\mathfrak{R}^{2}$ is a metric space with the metric $d$ defined by

$$
d\left(\bar{\xi}_{1}, \bar{\xi}_{2}\right)=\max \left\{\left|\xi_{1 \bar{\ell}}-\xi_{2 \bar{\ell}}\right|,\left|\xi_{1 \underline{\ell}}-\xi_{2 \underline{\ell}}\right|,\left|\xi_{1 \bar{r}}-\xi_{2 \bar{r}}\right|,\left|\xi_{1 \underline{\underline{r}}}-\xi_{2 \underline{\underline{r}}}\right|\right\} .
$$

Proof. The positivity and symmetry of $d$ are obvious. Let's show the triangle inequality. If $\bar{\xi}_{1}, \bar{\xi}_{2}$ and $\bar{\xi}_{3} \in \mathfrak{R}^{2}$, then

$$
\begin{aligned}
& d\left(\bar{\xi}_{1}, \bar{\xi}_{2}\right)=\max \left\{\left|\xi_{1 \bar{\ell}}-\xi_{2 \bar{\ell}}\right|,\left|\xi_{1 \underline{\ell}}-\xi_{2 \underline{\underline{\ell}}}\right|,\left|\xi_{1 \bar{r}}-\xi_{2 \bar{r}}\right|,\left|\xi_{1 \underline{r}}-\xi_{2 \underline{r}}\right|\right\}, \\
& d\left(\bar{\xi}_{3}, \bar{\xi}_{1}\right)+d\left(\bar{\xi}_{3}, \bar{\xi}_{2}\right)=\max \left\{\left|\xi_{3 \bar{\ell}}-\xi_{1 \bar{\ell}}\right|,\left|\xi_{3 \underline{\ell}}-\xi_{1 \underline{\ell}}\right|,\left|\xi_{3 \bar{r}}-\xi_{1 \bar{r}}\right|,\left|\xi_{3 \underline{\underline{r}}}-\xi_{1 \underline{\underline{r}}}\right|\right\} \\
& +\max \left\{\left|\xi_{3 \bar{\ell}}-\xi_{2 \bar{\ell}}\right|,\left|\xi_{3 \underline{\ell}}-\xi_{2 \underline{\ell}}\right|,\left|\xi_{3 \bar{r}}-\xi_{2 \bar{r}}\right|,\left|\xi_{3 \underline{r}}-\xi_{2 \underline{r}}\right|\right\} \\
& =\max \left\{\left|\xi_{3 \bar{\ell}}-\xi_{1 \bar{\ell}}\right|+\left|\xi_{3 \bar{\ell}}-\xi_{2 \bar{\ell}}\right|,\left|\xi_{3 \bar{\ell}}-\xi_{1 \bar{\ell}}\right|+\left|\xi_{3 \underline{\ell}}-\xi_{2 \underline{\ell}}\right|\right. \text {, } \\
& \left|\xi_{3 \bar{\ell}}-\xi_{1 \bar{\ell}}\right|+\left|\xi_{3 \bar{r}}-\xi_{2 \bar{r}}\right|,\left|\xi_{3 \bar{\ell}}-\xi_{1 \bar{\ell}}\right|+\left|\xi_{3 \underline{r}}-\xi_{2 \underline{r}}\right|, \\
& \left|\xi_{3 \underline{\ell}}-\xi_{1 \underline{\ell}}\right|+\left|\xi_{3 \bar{\ell}}-\xi_{2 \bar{\ell}}\right|,\left|\xi_{3 \underline{\ell}}-\xi_{1 \underline{\ell}}\right|+\left|\xi_{3 \underline{\ell}}-\xi_{2 \underline{\ell}}\right| \text {, } \\
& \left|\xi_{3 \underline{\underline{\ell}}}-\xi_{1 \underline{\ell}}\right|+\left|\xi_{3 \bar{r}}-\xi_{2 \bar{r}}\right|,\left|\xi_{3 \underline{\underline{\ell}}}-\xi_{1 \underline{\underline{\ell}}}\right|+\left|\xi_{3 \underline{\underline{r}}}-\xi_{2 \underline{\underline{r}}}\right| \text {, } \\
& \left|\xi_{3 \bar{r}}-\xi_{1 \bar{r}}\right|+\left|\xi_{3 \bar{\ell}}-\xi_{2 \bar{\ell}}\right|,\left|\xi_{3 \bar{r}}-\xi_{1 \bar{r}}\right|+\left|\xi_{3 \underline{\ell}}-\xi_{2 \underline{\ell}}\right| \text {, } \\
& \left|\xi_{3 \bar{r}}-\xi_{1 \bar{r}}\right|+\left|\xi_{3 \bar{r}}-\xi_{2 \bar{r}}\right|,\left|\xi_{3 \bar{r}}-\xi_{1 \bar{r}}\right|+\left|\xi_{3 \underline{r}}-\xi_{2 \underline{r}}\right| \text {, } \\
& \left|\xi_{3 \underline{r}}-\xi_{1 \underline{r}}\right|+\left|\xi_{3 \bar{\ell}}-\xi_{2 \bar{\ell}}\right|,\left|\xi_{3 \underline{r}}-\xi_{1 \underline{r}}\right|+\left|\xi_{3 \underline{\underline{\ell}}}-\xi_{2 \underline{\underline{\underline{r}}}}\right|, \\
& \left.\left|\xi_{3 \underline{r}}-\xi_{1 \underline{r}}\right|+\left|\xi_{3 \bar{r}}-\xi_{2 \bar{r}}\right|,\left|\xi_{3 \underline{\underline{r}}}-\xi_{1 \underline{r}}\right|+\left|\xi_{3 \underline{r}}-\xi_{2 \underline{r}}\right|\right\} \text {. }
\end{aligned}
$$

Now since

$$
\begin{aligned}
& \left|\xi_{1 \bar{\ell}}-\xi_{2 \bar{\ell}}\right| \leq\left|\xi_{3 \bar{\ell}}-\xi_{1 \bar{\ell}}\right|+\left|\xi_{3 \bar{\ell}}-\xi_{2 \bar{\ell}}\right|, \\
& \left|\xi_{1 \underline{\ell}}-\xi_{2 \underline{\ell}}\right| \leq\left|\xi_{3 \underline{\ell}}-\xi_{1 \underline{\ell}}\right|+\left|\xi_{3 \underline{\ell}}-\xi_{2 \underline{\ell}}\right|, \\
& \left|\xi_{1 \bar{r}}-\xi_{2 \bar{r}}\right| \leq\left|\xi_{3 \bar{r}}-\xi_{1 \bar{r}}\right|+\left|\xi_{3 \bar{r}}-\xi_{2 \bar{r}}\right|
\end{aligned}
$$

and

we have

$$
\left|\xi_{1 \underline{r}}-\xi_{2 \underline{r}}\right| \leq\left|\xi_{3 \underline{r}}-\xi_{1 \underline{r}}\right|+\left|\xi_{3 \underline{r}}-\xi_{2 \underline{r}}\right|
$$

$$
d\left(\bar{\xi}_{1}, \bar{\xi}_{2}\right) \leq d\left(\bar{\xi}_{3}, \bar{\xi}_{1}\right)+d\left(\bar{\xi}_{3}, \bar{\xi}_{2}\right)
$$


In the special case

$$
\bar{\xi}_{1}=\left(\left[\xi_{1 \underline{\underline{\ell}}}, \xi_{1 \underline{r}}\right],[0,0]\right)=\left[\xi_{1 \underline{\underline{\ell}}}, \xi_{1 \underline{r}}\right]
$$

and

$$
\bar{\xi}_{2}=\left(\left[\xi_{2 \underline{\underline{\ell}}}, \xi_{2 \underline{r}}\right],[0,0]\right)=\left[\xi_{2 \underline{\underline{\ell}}}, \xi_{2 \underline{r}}\right],
$$

we obtain the metric

$$
d\left(\bar{\xi}_{1}, \bar{\xi}_{2}\right)=\max \left\{\left|\xi_{1 \underline{\underline{\ell}}}-\xi_{2 \underline{\ell}}\right|,\left|\xi_{1 \underline{\underline{r}}}-\xi_{2 \underline{\underline{r}}}\right|\right\}
$$

defined on the set of all interval numbers.

Definition 2.1. A sequence of two dimensional interval numbers

$$
\left\{\bar{\xi}_{k}=\left(\left[\xi_{k \underline{\ell}}, \xi_{k \underline{r}}\right],\left[\xi_{k \bar{\ell}}, \xi_{k \bar{r}}\right]\right)\right\}_{k=1}^{\infty}
$$

is said to be bounded if there exists a real number $M>0$ such that $\left|\bar{\xi}_{k}\right| \leq M$ for all $k \in \mathbb{N}$.

Definition 2.2. The infinite sequence of two dimensional interval numbers

$$
\left\{\bar{\xi}_{k}=\left(\left[\xi_{k \underline{\ell}}, \xi_{k \underline{r}}\right],\left[\xi_{k \bar{\ell}}, \xi_{k \bar{r}}\right]\right)\right\}_{k=1}^{\infty}
$$

is said to be convergent to a bounded two dimensional interval number

$$
\bar{\xi}=\left(\left[\xi_{\underline{\underline{\ell}}}, \xi_{\underline{r}}\right],\left[\xi_{\bar{\ell}}, \xi_{\bar{r}}\right]\right)
$$

if for each $\epsilon>0$ there exists a positive integer $N$ such that $d\left(\bar{\xi}_{k}, \bar{\xi}\right)<\epsilon$ for all $k \geq N$. In this case, we write $\lim _{k \rightarrow \infty} \bar{\xi}_{k}=\bar{\xi}$.

Thus,

$$
\lim _{k \rightarrow \infty} \bar{\xi}_{k}=\bar{\xi} \Leftrightarrow \lim _{k \rightarrow \infty} \xi_{k \underline{\ell}}=\xi_{\underline{\ell}}, \quad \lim _{k \rightarrow \infty} \xi_{k \underline{r}}=\xi_{\underline{r}}, \quad \lim _{k \rightarrow \infty} \xi_{k \bar{\ell}}=\xi_{\bar{\ell}} \text { and } \lim _{k \rightarrow \infty} \xi_{k \bar{r}}=\xi_{\bar{r}} .
$$

As an example, let

$$
\left\{\bar{\xi}_{k}=\left(\left[\frac{1}{k+1}, \frac{k+1}{k}\right],\left[\frac{k}{k+1}, \frac{2 k}{k+1}\right]\right)\right\}
$$

then

$$
\lim _{k \rightarrow \infty} \bar{\xi}_{k}=([0,1],[1,2]) .
$$

A two dimensional interval sequence $\left\{\bar{\xi}_{k}\right\}$ is nested if $\bar{\xi}_{k+1} \subseteq \bar{\xi}_{k}$ for all $k$.

Every nested two dimensional interval sequence $\left\{\bar{\xi}_{k}\right\}$ converges and has the limit $\bigcap_{k=1}^{\infty} \bar{\xi}_{k}$.

For a sequence of $\left(x_{k}\right)$ of real numbers, it is easy to see that

$$
\lim _{k \rightarrow \infty} x_{k}=x \Leftrightarrow \lim _{k \rightarrow \infty}\left|x_{k}-x\right|=0
$$

This can be extended to the sequences of two dimensional intervals.

Theorem 2.2. Let the sequence of two dimensional intervals

$$
\left\{\bar{\xi}_{k}=\left(\left[\xi_{k \underline{\ell}}, \xi_{k \underline{r}}\right],\left[\xi_{k \bar{\ell}}, \xi_{k \bar{r}}\right]\right)\right\}_{k=1}^{\infty}
$$

be convergent to a bounded two dimensional interval

$$
\bar{\xi}=\left(\left[\xi_{\underline{\underline{\ell}}}, \xi_{\underline{r}}\right],\left[\xi_{\bar{\ell}}, \xi_{\bar{r}}\right]\right),
$$

then

$$
\lim _{k \rightarrow \infty} \bar{\xi}_{k}=\bar{\xi} \Leftrightarrow \lim _{k \rightarrow \infty}\left|\bar{\xi}_{k}-\bar{\xi}\right|=0
$$


Proof.

$$
\begin{aligned}
\lim _{k \rightarrow \infty} \bar{\xi}_{k}=\bar{\xi} & \Leftrightarrow \lim _{k \rightarrow \infty} \xi_{k \underline{\ell}}=\xi_{\underline{\ell}}, \lim _{k \rightarrow \infty} \xi_{k \underline{r}}=\xi_{\underline{r}}, \lim _{k \rightarrow \infty} \xi_{k \bar{\ell}}=\xi_{\bar{\ell}} \text { and } \lim _{k \rightarrow \infty} \xi_{k \bar{r}}=\xi_{\bar{r}} \\
& \Leftrightarrow \lim _{k \rightarrow \infty}\left|\xi_{k \underline{\ell}}-\xi_{\underline{\ell}}\right|=0, \lim _{k \rightarrow \infty}\left|\xi_{k \underline{r}}-\xi_{\underline{r}}\right|=0, \lim _{k \rightarrow \infty}\left|\xi_{k \bar{\ell}}-\xi_{\bar{\ell}}\right|=0 \text { and } \lim _{k \rightarrow \infty}\left|\xi_{k \bar{r}}-\xi_{\bar{r}}\right|=0 \\
& \Leftrightarrow \max \left\{\lim _{k \rightarrow \infty}\left|\xi_{k \underline{\underline{\ell}}}-\xi_{\underline{\ell}}\right|, \lim _{k \rightarrow \infty}\left|\xi_{k \underline{r}}-\xi_{\underline{r}}\right|, \lim _{k \rightarrow \infty}\left|\xi_{k \bar{\ell}}-\xi_{\bar{\ell}}\right|, \lim _{k \rightarrow \infty}\left|\xi_{k \bar{r}}-\xi_{\bar{r}}\right|\right\}=0 \\
& \Leftrightarrow \lim _{k \rightarrow \infty} \max \left\{\left|\xi_{k \underline{\underline{\ell}}}-\xi_{\underline{\underline{\ell}}}\right|,\left|\xi_{k \underline{r}}-\xi_{\underline{r}}\right|,\left|\xi_{k \bar{l}}-\xi_{\bar{l}}\right|,\left|\xi_{k \bar{r}}-\xi_{\bar{r}}\right|\right\}=0 \\
& \Leftrightarrow \lim _{k \rightarrow \infty}\left|\bar{\xi}_{k}-\bar{\xi}\right|=0 .
\end{aligned}
$$

Definition 2.3. The infinite sequence of two dimensional interval numbers

$$
\left\{\bar{\xi}_{k}=\left(\left[\xi_{k \underline{\ell}}, \xi_{k \underline{r}}\right],\left[\xi_{k \bar{\ell}}, \xi_{k \bar{r}}\right]\right)\right\}_{k=1}^{\infty}
$$

is said to be two dimensional interval Cauchy sequence if for each $\epsilon>0$ there exists a positive integer $N$ such that $d\left(\bar{\xi}_{i}, \bar{\xi}_{j}\right)<\epsilon$ for all $i, j \geq N$.

An $n$-dimensional two dimensional interval vector is an ordered $n$-tuples of two dimensional intervals.

$$
\bar{\xi}=\left[\bar{\xi}_{1}, \bar{\xi}_{2}, \ldots, \bar{\xi}_{n}\right]^{T}=\left[\left(\left[\xi_{1 \underline{\ell}}, \xi_{1 \underline{r}}\right],\left[\xi_{1 \bar{\ell}}, \xi_{1 \bar{r}}\right]\right),\left(\left[\xi_{2 \underline{\ell}}, \xi_{2 \underline{r}}\right],\left[\xi_{2 \bar{\ell}}, \xi_{2 \bar{r}}\right]\right), \ldots,\left(\left[\xi_{n \underline{\ell}}, \xi_{n \underline{r}}\right],\left[\xi_{n \bar{\ell}}, \xi_{n \bar{r}}\right]\right)\right]^{T} .
$$

If $|\bar{\xi}|=0$, then $\bar{\xi}$ is said to be a zero two dimensional interval and $\bar{\xi}$ must be a degenerate interval $\overline{0}=([0,0],[0,0])$.

Some well-known inequalities can be extended to the two dimensional interval vectors as in the following two lemmas with absolute value defined in (1.1).

Lemma 2.1. Let $\bar{\xi}_{1}$ and $\bar{\xi}_{2}$ be two dimensional intervals, then

$$
\left|\bar{\xi}_{1}+\bar{\xi}_{2}\right| \leq\left|\bar{\xi}_{1}\right|+\left|\bar{\xi}_{2}\right|
$$

Proof.

$$
\begin{aligned}
\left|\bar{\xi}_{1}+\bar{\xi}_{2}\right| & =\max \left\{\left|\xi_{1 i \underline{\underline{\ell}}}+\xi_{2 i \underline{\ell}}\right|,\left|\xi_{1 i \underline{\underline{r}}}+\xi_{2 i \underline{\underline{r}}}\right|,\left|\xi_{1 i \bar{\ell}}+\xi_{2 i \bar{\ell}}\right|,\left|\xi_{1 i \bar{r}}+\xi_{2 i \bar{r}}\right|\right\} \\
& \leq \max \left\{\left|\xi_{1 i \underline{\ell}}\right|+\left|\xi_{2 i \underline{\ell}}\right|,\left|\xi_{1 i \underline{r}}\right|+\left|\xi_{2 i \underline{\underline{r}}}\right|,\left|\xi_{1 i \bar{\ell}}\right|+\left|\xi_{2 i \bar{\ell}}\right|,\left|\xi_{1 i \bar{r}}\right|+\left|\xi_{2 i \bar{r}}\right|\right\} \\
& =\max \left\{\left|\xi_{1 i \underline{\underline{\ell}}}\right|,\left|\xi_{1 i \underline{\underline{r}}}\right|,\left|\xi_{1 i \bar{\ell}}\right|,\left|\xi_{1 i \bar{r}}\right|\right\}+\max \left\{\left|\xi_{2 i \underline{\underline{r}}}\right|,\left|\xi_{2 i \underline{\underline{r}}}\right|,\left|\xi_{2 i \bar{\ell}}\right|,\left|\xi_{2 i \bar{r}}\right|\right\} \\
& =\left|\bar{\xi}_{1}\right|+\left|\bar{\xi}_{2}\right|
\end{aligned}
$$

The inner product of two dimensional interval vectors is defined trough the two dimensional interval multiplication as follows:

$$
\begin{aligned}
& <\bar{\xi}_{1}, \bar{\xi}_{2}>=\sum_{i=1}^{n} \bar{\xi}_{1 i} \bar{\xi}_{2 i} \\
& =\sum_{i=1}^{n}\left(\left[\bar{\xi}_{1 i \underline{\ell}}, \bar{\xi}_{1 i \underline{\underline{2}}}\right],\left[\bar{\xi}_{1 i \bar{\ell}}, \bar{\xi}_{1 i \bar{r}}\right]\right)\left(\left[\bar{\xi}_{2 i \underline{\ell}}, \bar{\xi}_{2 i \underline{ }}\right],\left[\bar{\xi}_{2 i \bar{\ell}}, \bar{\xi}_{2 i \bar{r}}\right]\right)
\end{aligned}
$$

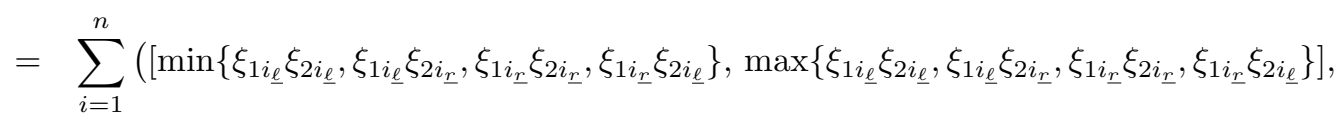

$$
\begin{aligned}
& \left.\left[\min \left\{\xi_{1 i_{\bar{\ell}}} \xi_{2 i_{\bar{\ell}}}, \xi_{1 i_{\bar{\ell}}} \xi_{2 i_{\bar{r}}}, \xi_{1 i_{\bar{r}}} \xi_{2 \bar{r}}, \xi_{1 \bar{r}} \xi_{2 \bar{\ell}}\right\}, \max \left\{\xi_{1 \bar{\ell}} \xi_{2 i_{\bar{\ell}}}, \xi_{1 i_{\bar{\ell}}} \xi_{2 i_{\bar{r}}}, \xi_{1 i_{\bar{r}}} \xi_{2 i_{\bar{r}}}, \xi_{1 i_{\bar{r}}} \xi_{2 i_{\bar{\ell}}}\right\}\right]\right) .
\end{aligned}
$$

Lemma 2.2. Let $\bar{\xi}_{1}$ and $\bar{\xi}_{2}$ be two dimensional interval vectors in $\left(\mathfrak{R}^{2}\right)^{n}$. Then

$$
\left|<\bar{\xi}_{1}, \bar{\xi}_{2}>\right| \leq\left|<\bar{\xi}_{1}, \bar{\xi}_{1}>\right|^{\frac{1}{2}}\left|<\bar{\xi}_{2}, \bar{\xi}_{2}>\right|^{\frac{1}{2}}
$$


Proof. Let $\alpha$ be any real number and the following inequality is always true

$$
\left|<\alpha \bar{\xi}_{1}+\bar{\xi}_{2}, \alpha \bar{\xi}_{1}+\bar{\xi}_{2}>\right| \geq 0
$$

Then

$$
\begin{aligned}
0 & \leq\left|<\alpha \bar{\xi}_{1}+\bar{\xi}_{2}, \alpha \bar{\xi}_{1}+\bar{\xi}_{2}>\right| \\
& \leq\left|<\alpha^{2} \bar{\xi}_{1}, \bar{\xi}_{2}>+2 \alpha<\bar{\xi}_{1}, \bar{\xi}_{2}>+<\bar{\xi}_{1}, \bar{\xi}_{2}>\right| \\
& \leq \alpha^{2}\left|<\bar{\xi}_{1}, \bar{\xi}_{2}>+2\right| \alpha||<\bar{\xi}_{1}, \bar{\xi}_{2}>|+|<\bar{\xi}_{1}, \bar{\xi}_{2}>\mid
\end{aligned}
$$

The right hand side of the last inequality is a quadratic form for $|\alpha|$ and it is always greater than or equal to 0 . Therefore

$$
\left|<\bar{\xi}_{1}, \bar{\xi}_{2}>\right|^{2}-\left|<\bar{\xi}_{1}, \bar{\xi}_{1}>\right|\left|<\bar{\xi}_{2}, \bar{\xi}_{2}>\right| \leq 0,
$$

thus

$$
\left|<\bar{\xi}_{1}, \bar{\xi}_{2}>\right| \leq\left|<\bar{\xi}_{1}, \bar{\xi}_{1}>\right|^{\frac{1}{2}}\left|<\bar{\xi}_{2}, \bar{\xi}_{2}>\right|^{\frac{1}{2}}
$$

Let $\left(\Re^{2}\right)^{n}$ be the set of two dimensional interval vectors. Some properties of the classical vector norms can be extended to the two dimensional interval vectors. The max-norm of a two dimensional interval vector on $\left(\mathfrak{R}^{2}\right)^{n}$ is a non-negative valued function

$$
\|\cdot\|:\left(\mathfrak{R}^{2}\right)^{n} \rightarrow \mathbb{R}^{+} \cup\{0\}
$$

that satisfies following properties:

(1) $\forall \bar{\xi} \in\left(\mathfrak{R}^{2}\right)^{n}-\{\overline{0}\},\|\bar{\xi}\|>0$,

(2) $\forall \bar{\xi} \in\left(\mathfrak{R}^{2}\right)^{n}$ and $\alpha \in \mathbb{R},\|\alpha \bar{\xi}\|=|\alpha|\|\bar{\xi}\|$,

(3) $\forall \bar{\xi}, \bar{\zeta} \in\left(\Re^{2}\right)^{n},\|\bar{\xi}+\bar{\zeta}\| \leq\|\bar{\xi}\|+\|\bar{\zeta}\|$.

Theorem 2.3. $\left(\mathfrak{R}^{2}\right)^{n}$ with the following norm is a normed space

$$
\|\bar{\xi}\|=\left(\sum_{i=1}^{n}\left|\bar{\xi}_{i}\right|^{\frac{1}{2}}\right)^{2}
$$

Proof. If $\bar{\xi} \neq \overline{0}$, then $\|\bar{\xi}\|>0$.

$$
\begin{aligned}
\|\alpha \bar{\xi}\| & =\left(\sum_{i=1}^{n}\left(\max \left\{\left|\alpha \xi_{i_{\underline{\underline{\varepsilon}}}}\right|,\left|\alpha \xi_{i_{\underline{\underline{r}}}}\right|,\left|\alpha \xi_{i_{\bar{\ell}}}\right|,\left|\alpha \xi_{i_{\bar{r}}}\right|\right\}\right)^{2}\right)^{\frac{1}{2}} \\
& =|\alpha|\left(\sum_{i=1}^{n}\left(\max \left\{\left|\xi_{i_{\underline{\underline{\ell}}}}\right|,\left|\xi_{i_{\underline{\underline{r}}}}\right|,\left|\xi_{i_{\bar{\ell}}}\right|,\left|\xi_{i_{\bar{r}}}\right|\right\}\right)^{2}\right)^{\frac{1}{2}} \\
& =|\alpha|\|\bar{\xi}\| .
\end{aligned}
$$




$$
\begin{aligned}
& \|\bar{\xi}+\bar{\zeta}\|=\left(\sum_{i=1}^{n}\left|\bar{\xi}_{i}+\bar{\zeta}_{i}\right|^{2}\right)^{\frac{1}{2}} \\
& =\left(\sum_{i=1}^{n}\left(\max \left\{\left|\xi_{\underline{i_{\underline{\ell}}}}+\zeta_{\underline{\underline{\ell}}_{\underline{\underline{\ell}}}}\right|,\left|\xi_{i_{\underline{\underline{r}}}}+\zeta_{i_{\underline{\underline{r}}}}\right|,\left|\xi_{i_{\bar{\ell}}}+\zeta_{i_{\bar{\ell}}}\right|,\left|\xi_{i_{\bar{r}}}+\zeta_{i_{\bar{r}}}\right|\right\}\right)^{2}\right)^{\frac{1}{2}} \\
& =\left(\sum_{i=1}^{n}\left|\bar{\xi}_{i_{m_{i}}}+\bar{\zeta}_{i_{m_{i}}}\right|^{2}\right)^{\frac{1}{2}} \quad\left(m_{i}=\underline{\ell}, \bar{\ell}, \underline{r} \text { or } \bar{r}\right) \\
& =\left\|\xi_{m}+\zeta_{m}\right\|_{2} \quad \text { (ordinary vector norm) } \\
& \leq\left\|\xi_{m}\right\|_{2}+\left\|\zeta_{m}\right\|_{2} \\
& =\left(\sum_{i=1}^{n}\left|\bar{\xi}_{i_{m_{i}}}\right|^{2}\right)^{\frac{1}{2}}+\left(\sum_{i=1}^{n}\left|\bar{\zeta}_{i_{m_{i}}}\right|^{2}\right)^{\frac{1}{2}} \\
& \leq\left(\sum_{i=1}^{n} \max \left\{\left|\xi_{i_{\underline{\underline{\ell}}}}\right|^{2},\left|\xi_{i_{\underline{\underline{r}}}}\right|^{2},\left|\xi_{\overline{\bar{\ell}}_{\bar{\ell}}}\right|^{2},\left|\xi_{i_{\bar{\tau}}}\right|^{2}\right\}\right)^{\frac{1}{2}} \\
& +\left(\sum_{i=1}^{n} \max \left\{\left|\zeta_{i_{\underline{\underline{\ell}}}}\right|^{2},\left|\zeta_{i_{\underline{r}}}\right|^{2},\left|\zeta_{i_{\bar{\ell}}}\right|^{2},\left|\zeta_{i_{\bar{r}}}\right|^{2}\right\}\right)^{\frac{1}{2}} \\
& =\|\bar{\xi}\|+\|\bar{\zeta}\|
\end{aligned}
$$

where $\xi_{m}=\left[\xi_{m_{m_{1}}}, \xi_{2_{m_{2}}}, \ldots, \xi_{n_{m_{n}}}\right]^{T}, \zeta_{m}=\left[\zeta_{1_{m_{1}}}, \zeta_{2_{m_{2}}}, \ldots, \zeta_{n_{m_{n}}}\right]^{T}$. Thus, axioms of the norm function are hold and the proof is completed.

Observe that

$$
\|\bar{\xi}\|_{1}=\sum_{i=1}^{n}\left|\bar{\xi}_{i}\right|
$$

and

$$
\|\bar{\xi}\|_{\infty}=\max _{i}\left\{\max \left\{\left|\xi_{i \underline{\ell}}\right|,\left|\xi_{i \underline{\underline{r}}}\right|,\left|\xi_{i \bar{\ell}}\right|,\left|\xi_{i \bar{r}}\right|\right\}\right\}
$$

are the other norms on $\left(\mathfrak{R}^{2}\right)^{n}$.

\section{Statistical Convergence}

Statistical convergence of sequences of numbers was introduced by Fast [4]. In [13], Schoenberg established some basic properties of statistical convergence and also studied the concept as a summability method.

A sequence $\left(x_{k}\right)$ is said to be statistically convergent to the number $\ell$ if for every $\epsilon>0$

$$
\lim _{n \rightarrow \infty} \frac{1}{n}\left|\left\{k \leq n:\left|x_{k}-\ell\right| \geq \epsilon\right\}\right|=0 .
$$

In this case, we write $s t-\lim x_{k}=\ell \cdot \lim x_{k}=\ell$ implies $s t-\lim x_{k}=\ell$, so statistical convergence may be considered as a regular summability method. This was observed in [13] along with the fact that the statistical limit is a linear functional on some sequence spaces.

In [5], Fridy proved that if $\left(x_{k}\right)$ is a statistically convergent sequence, then there is a convergent sequence $\left(y_{k}\right)$ such that

$$
\lim _{n \rightarrow \infty} \frac{1}{n}\left|\left\{k \leq n: x_{k} \neq y_{k}\right\}\right|=0 .
$$

Now we will give definitions of statistical convergence, statistically Cauchy and Cesàro summability for the two dimensional interval numbers and we will get the relationships between them. 
Definition 3.1. The infinite sequence of two dimensional interval numbers

$$
\left\{\bar{\xi}_{k}=\left(\left[\xi_{k \underline{\ell}}, \xi_{k \underline{r}}\right],\left[\xi_{k \bar{\ell}}, \xi_{k \bar{r}}\right]\right)\right\}_{k=1}^{\infty}
$$

is said to be statistically convergent to a bounded two dimensional interval number

$$
\bar{\xi}=\left(\left[\xi_{\underline{\underline{\ell}}}, \xi_{\underline{r}}\right],\left[\xi_{\bar{\ell}}, \xi_{\bar{r}}\right]\right)
$$

if for each $\epsilon>0$

$$
\lim _{n \rightarrow \infty} \frac{1}{n}\left|\left\{k \leq n: d\left(\bar{\xi}_{k}, \bar{\xi}\right) \geq \epsilon\right\}\right|=0,
$$

where the vertical bars denote the number of elements in the enclosed set. In this case, we write $s t-\lim _{k \rightarrow \infty} \bar{\xi}_{k}=\bar{\xi}_{\text {. }}$

Thus,

$$
s t-\lim _{k \rightarrow \infty} \bar{\xi}_{k}=\bar{\xi} \Leftrightarrow s t-\lim _{k \rightarrow \infty} \xi_{k \underline{\ell}}=\xi_{\underline{\ell}}, s t-\lim _{k \rightarrow \infty} \xi_{k \underline{r}}=\xi_{\underline{r}}, \quad s t-\lim _{k \rightarrow \infty} \xi_{k \bar{\ell}}=\xi_{\bar{\ell}} \text { and } s t-\lim _{k \rightarrow \infty} \xi_{k \bar{r}}=\xi_{\bar{r} .}
$$

Statistical convergence is a natural generalization of ordinary convergence. If $\lim _{k \rightarrow \infty} \bar{\xi}_{k}=\bar{\xi}$, then $s t-\lim _{k \rightarrow \infty} \bar{\xi}_{k}=\bar{\xi}$. The converse does not hold in general. For example, let $\left(\bar{\xi}_{k}\right)$ be following sequence:

$$
\bar{\xi}_{k}:= \begin{cases}([k, k+1],[k+1, k+2]) & , \text { if and } k \text { is a square integer } \\ ([0,0],[0,0]) & , \text { otherwise. }\end{cases}
$$

This sequence of two dimensional interval numbers is not convergent. But since

$$
\frac{1}{n} \mid\left\{k \leq n: d\left(\bar{\xi}_{k},([0,0],[0,0])\right) \geq \epsilon \mid \leq \frac{\sqrt{n}}{n},\right.
$$

this sequence is statistically convergent to the $([0,0],[0,0])$.

Definition 3.2. The infinite sequence of two dimensional interval numbers

$$
\left\{\bar{\xi}_{k}=\left(\left[\xi_{k \underline{\ell}}, \xi_{k \underline{r}}\right],\left[\xi_{k \bar{\ell}}, \xi_{k \bar{r}}\right]\right)\right\}_{k=1}^{\infty}
$$

is said to be statistically Cauchy sequence if for each $\epsilon>0$ there exists a positive integer $\mathrm{N}$ such that

$$
\lim _{n \rightarrow \infty} \frac{1}{n}\left|\left\{k \leq n: d\left(\bar{\xi}_{k}, \bar{\xi}_{N}\right) \geq \epsilon\right\}\right|=0 .
$$

Theorem 3.1. The following statements are equivalent:

$i .\left(\bar{\xi}_{k}\right)$ is a statistically convergent sequence,

ii. $\left(\bar{\xi}_{k}\right)$ is a statistically Cauchy sequence,

iii. $\left(\bar{\xi}_{k}\right)$ is a sequence for which there is a convergent sequence $\left(\bar{\zeta}_{k}\right)$ such that $\lim _{n \rightarrow \infty} \frac{1}{n}\left|\left\{k \leq n: \bar{\xi}_{k} \neq \bar{\zeta}_{k}\right\}\right|=0$.

The theorem can be proved using techniques similar to those in [5], so we omit it.

Definition 3.3. We say that $\left(\bar{\xi}_{k}\right)$ is Cesàro summable to $\bar{\xi}$ if

$$
\lim _{n \rightarrow \infty} \frac{1}{n} \sum_{k=1}^{n} d\left(\bar{\xi}_{k}, \bar{\xi}\right)=0 .
$$

\section{Theorem 3.2. .}

i. If $\left(\bar{\xi}_{k}\right)$ is Cesàro summable to $\bar{\xi}$, then it is statistically convergent to $\bar{\xi}$,

ii. If $\left(\bar{\xi}_{k}\right)$ is bounded and statistically convergent to $\bar{\xi}$, then it is Cesàro summable to $\bar{\xi}$.

The theorem can be proved using techniques similar to those in [13], so we omit it. 


\section{Funding}

There is no funding for this work.

\section{Availability of data and materials}

Not applicable.

\section{Competing interests}

The authors declare that they have no competing interests.

\section{Author's contributions}

All authors contributed equally to the writing of this paper. All authors read and approved the final manuscript.

\section{References}

[1] Chiao, K.-P.: Fundamental properties of interval vector max-norm. Tamsui Oxf. J. Math. Sci. 18 (2), 219-233 (2002).

[2] Dwyer, P. S.: Linear Computations. Wiley, New York (1951).

[3] Dwyer, P. S.: Errors of matrix computation, simultaneous equations and eigenvalues, National Bureu of Standarts. Applied Mathematics Series. 29, 49-58 (1953).

[4] Fast, H.: Sur la convergence statistique. Colloq. Math. 2, 241-244 (1951).

[5] Fridy, J. A.: On statistical convergence. Analysis (Munich). 5 (4), 301-314 (1985).

[6] Gürdal, M., Huban, M. B.: On I-convergence of double sequences in the Topology induced by random 2-norms. Matematicki Vesnik. 66 (1), 73-83 (2014).

[7] Gürdal, M., Şahiner, A.: Extremal I-limit points of double sequences. Appl. Math. E-Notes. 8, 131-137 (2008).

[8] Markov, S. M.: Extended interval arithmetic involving infinite intervals. Math. Balkanica, New Series. 6 (3), 269-304 (1992).

[9] Markov, S.: On directed interval arithmetic and its applications. J.UCS. 1 (7), 514-526 (1995).

[10] Markov, S.: Quasilinear spaces and their relation to vector spaces. Electronic Journal on Mathematics of Computation. $2(1), 1-21(2005)$.

[11] Moore, R. E.: Automatic error analysis in digital computation. Lockheed Missiles and Space Company, Technical Report LMSD-448421. California (1959).

[12] Moore, R. E., Yang, C. T.: Interval analysis I. Lockheed Missiles and Space Division, Technical Report LMSD288139. California (1960).

[13] Schoenberg, I. J.: The integrability of certain functions and related summability methods. Amer. Math. Monthly. 66 (5), 361-375 (1959).

[14] Sunaga, T.: Theory of an interval algebra and its application to numerical analysis. In: Research Association of Applied Geometry Memoirs II (pp. 29-46). Gakujutsu Bunken Fukyu-kai, Tokyo (1958).

[15] Şengönül, M., Eryilmaz, A.: On the sequence spaces of interval numbers. Thai J. Math. 8 (3), 503-510 (2010). 


\section{Affiliations}

FATIH NURAY

ADDRESS: Afyon Kocatepe University, Department of Mathematics, 03200, Afyonkarahisar-Turkey. E-MAIL: fnuray@aku.edu.tr ORCID ID: 0000-0003-0160-4001

UĞUR ULUSU

ADDRESS: Sivas Cumhuriyet University, 58140, Sivas-Turkey.

E-MAIL: ugurulusu@cumhuriyet.edu.tr

ORCID ID: 0000-0001-7658-6114

ERDINÇ DÜNDAR

ADDRESS: Afyon Kocatepe University, Department of Mathematics, 03200, Afyonkarahisar-Turkey. E-MAIL: edundar@aku.edu.tr

ORCID ID: 0000-0002-0545-7486 